\title{
National Analysis of Short-Term Outcomes and Volume-Outcome Relationships for Transcatheter Aortic Valve Replacement in the Era of Commercialization
}

\author{
Andreas R. de Biasi Subroto Paul Abu Nasar Leonard N. Girardi \\ Arash Salemi \\ Department of Cardiothoracic Surgery, Weill Cornell Medical College, New York-Presbyterian Hospital, \\ New York, N.Y., USA
}

\section{Key Words}

Aortic stenosis - Cardiac surgery - Transcatheter aortic valve replacement

\begin{abstract}
Objectives: We queried the 2012 National Inpatient Sample in order to (1) further describe the short-term outcomes for transcatheter aortic valve replacement (TAVR) and (2) characterize possible volume-outcome relationships and other prognostic factors for this procedure. Methods: Demographics and inhospital outcomes were tabulated for all patients, as were hospital characteristics and procedural-volume data for all centers at which patients underwent TAVR. Logistic regression analyses were performed to identify independent risk factors for mortality or morbidity. Results: 7,635 patients aged $\geq 18$ years received TAVR during the study period; $84.5 \%$ ( $n=6,450$ ) underwent transfemoral TAVR and the rest were treated transapically. The median age was 83 years (IQR 77-88 years) and cardiovascular comorbidities were widespread. Overall inhospital mortality was $5.0 \%(n=380)$, and $1.4 \%(n=105)$ of the patients experienced a stroke. All-cause procedure-related morbidity was $24.7 \%(n=1,885)$. Annual hospital TAVR volume did not predict inhospital mortality or morbidity (OR $1.00,95 \% \mathrm{Cl} 0.99-$
\end{abstract}

$1.00, \mathrm{p}=0.111$ and OR $1.00,95 \% \mathrm{Cl} 0.99-1.00, \mathrm{p}=0.947$, respectively). Conclusions: Our analysis helps to confirm the short-term safety profile of TAVR and further demonstrates that inhospital outcomes have remained acceptable as this procedure has become commercialized.

(c) 2015 S. Karger AG, Basel

\section{Introduction}

Transcatheter aortic valve replacement (TAVR) has become a viable treatment option for inoperable and high-risk patients with severe, symptomatic aortic stenosis [1-7]. The evidence base for the emergence of TAVR stems mainly from clinical trials with inclusion and exclusion criteria that may no longer be reflective of the 'real-world' experience with this technique now that it has been approved by the USA FDA for both the transfemoral (TF) and transapical (TA) approaches $[8,9]$. To date, the largest postapproval studies on TAVR in the USA have come from the STS/ACC TVT (Society of Thoracic Surgeons/American College of Cardiology Transcatheter Valve Therapy) Registry, which has demonstrated inhospital and 30-day mortality rates for this procedure of $5.2-5.5 \%$ and $7.0-7.6 \%$, respectively $[5,10]$.

\section{KARGER}

E-Mail karger@karger.com

www.karger.com/crd
(C) 2015 S. Karger AG, Basel

0008-6312/15/1331-0058\$39.50/0 
Despite these early reports and in light of the rapid growth of TAVR, short-term safety and efficacy questions surrounding the mainstream use of this therapy remain incompletely answered. Moreover, as TAVR has moved beyond the confines of regimented clinical trials involving highly experienced centers, little has been done to examine the influence that hospital experience may have on the short-term outcomes of patients undergoing TAVR.

We therefore queried the National Inpatient Sample (NIS) in order to (1) further describe the short-term (i.e. inhospital) outcomes for TAVR and (2) characterize possible volume-outcome relationships and other prognostic factors for this procedure during the era of its commercialization.

\section{Methods}

\section{Data Source}

The Healthcare Cost and Utilization Project (HCUP), with sponsoring from the Agency for Healthcare Research and Quality, has maintained the NIS since 1988. This database can be used to analyze national trends in health care utilization, access and outcomes, among other metrics [11-15]. The NIS contains data on more than 7 million hospital stays each year from approximately 1,000 nongovernmental institutions and, when weighted, it estimates roughly 40 million hospitalizations, making it the largest publicly available all-payer inpatient database in the USA [11]. Data contained within the NIS include patient and hospital demographics, admission and treating diagnoses, inpatient procedures, inhospital mortality, length of stay and hospital charges as well as discharge status. The NIS dataset has numerous internal quality measures and is validated by the HCUP through comparisons to other similar databases [11]. This study was approved by the Institutional Review Board of Weill Cornell Medical College (protocol No. EXE 2011-040) and conforms to the data-use agreement for the NIS from the HCUP.

\section{Study Cohort}

The study cohort consisted of patients $\geq 18$ years of age who underwent either TF or TA TAVR as their principal procedure upon admission to an NIS-participating hospital in 2012 - the only full year for which TAVR data are currently available from the NIS. These patients - and their corresponding data of interest - were identified within the NIS using ICD-9-CM diagnosis and procedure codes. A full listing of the codes used for query can be found in online supplementary appendix table 1 (for all online suppl. material, see www.karger.com/doi/10.1159/000440694).

\section{Variables and Outcomes}

Demographic data were obtained for each patient identified for inclusion: age at admission, gender, race and insurance status; relevant comorbidities were also collected. Hospital characteristics (i.e. size, setting and region) as well as procedural volume data were obtained for all of the centers at which cohort patients underwent TAVR. Operative details, i.e. cardiopulmonary bypass (CPB) and the use of intra-aortic balloon pump (IABP), were also recorded.
The outcomes of this study included short-term (i.e. inhospital) mortality and morbidity (including procedure-related, cardiovascular, pulmonary, and infectious complications), length of stay and discharge status. Comorbidities and inhospital complications were identified by means of the respective ICD-9-CM diagnosis or procedure codes for the patients (online suppl. appendix table 1); comorbidities were recorded using the NIS Comorbidity software v3.7 [16].

\section{Study Objectives and Statistical Analyses}

The primary objective of this study was to describe the shortterm (i.e. inhospital) mortality and morbidity for TAVR performed in the era during which they were commercialized. Secondarily, we sought to determine the risk factors for these outcomes and, in so doing, investigated what effects the hospital experience, measured by annual procedural volumes, may have had on short-term mortality and morbidity.

Of note, in assessing for such possible volume-outcome relationships, hospitals were categorized into groups based on their 2012 TAVR experience. This grouping was defined using the Centers for Medicare and Medicaid Services' (CMS) recent National Coverage Determination for TAVR as a guide; in this document, the CMS states that in order for these operations to be reimbursed by the federal government, TAVRs are only to be performed by heart teams (i.e. centers) completing at least 20 such procedures annually [17]. We therefore defined the lowest-volume group as hospitals that performed $<20$ TAVRs in 2012, the next included hospitals performing the minimum CMS requirement up to double the CMS-mandated volume (i.e. 20-39 TAVRs), the third encompassed centers performing 2-3 times the CMS requirement (i.e. 40-59 TAVRs) and the highest-volume group was set as those hospitals performing at least triple the CMS-mandated number of procedures (i.e. $\geq 60$ TAVRs).

SUDAAN v9.0.1 (Research Triangle Institute, Research Triangle Park, N.C., USA) was used to convert raw counts generated from the NIS database into weighted counts that were used to generate national estimates. All results presented here, including all statistical analyses, reflect these weighted data (i.e. the national estimates). For categorical data, the $\chi^{2}$ test and analysis of variance for continuous data were used to test for statistical significance. All $\mathrm{p}$ values were 2 -sided with statistical significance evaluated at the 0.05 alpha level. Logistic regression analysis was used to determine clinical characteristics that were independent risk factors for mortality or morbidity. Univariable predictors of mortality or morbidity with a $p$ value $\leq 0.200$ that were thought to be of clinical significance a priori were entered into multivariable models. Colinearity between independent variables was assessed using variance influence factors. No variables tested were found to be colinear. All statistical analyses were performed using SPSS v17.0 (SPSS Inc., Chicago, Ill., USA) and SUDAAN v9.0.1.

\section{Results}

\section{Patient Demographics and Comorbidities}

A total of 7,635 patients underwent TAVR during the 1 -year study period; table 1 summarizes the demographics and comorbidities. Overall, the median age at the time of TAVR was 83 years (IQR 77-88 years) and $51.1 \%(\mathrm{n}=$ 
Table 1. Patient demographics and comorbidities

\begin{tabular}{|c|c|c|c|c|}
\hline & $\begin{array}{l}\text { Total } \\
(\mathrm{n}=7,635)\end{array}$ & $\begin{array}{l}\text { TF } \\
(n=6,450)\end{array}$ & $\begin{array}{l}\text { TA } \\
(n=1,185)\end{array}$ & $\mathrm{p}$ value $\mathrm{e}^{\mathrm{a}}$ \\
\hline \multicolumn{5}{|l|}{ Demographics } \\
\hline Median age, years (IQR) & $83(77-8)$ & $84(77-88)$ & $81(74-86)$ & $<0.001$ \\
\hline Gender & & & & 0.089 \\
\hline Male & $3,900(51.1)$ & $3,355(52.0)$ & $545(46.0)$ & \\
\hline Female & $3,735(48.9)$ & $3,095(48.0)$ & $640(54.0)$ & \\
\hline Race & & & & 0.116 \\
\hline White & $6,040(84.1)$ & $5,170(84.8)$ & $870(80.2)$ & \\
\hline Non-White & $1,145(15.9)$ & $930(15.2)$ & $215(19.8)$ & \\
\hline Insurance & & & & 0.019 \\
\hline Medicare & $6,845(89.7)$ & 5,865 (90.9) & $980(82.7)$ & \\
\hline Medicaid & $80(1.0)$ & $60(0.9)$ & $20(1.7)$ & \\
\hline Commercial & $580(7.6)$ & $435(6.7)$ & $145(12.2)$ & \\
\hline Other (uninsured) & $130(1.7)$ & $90(1.4)$ & $40(3.4)$ & \\
\hline \multicolumn{5}{|l|}{ Comorbidities } \\
\hline Stroke/TIA & $965(12.6)$ & $810(12.6)$ & $155(13.1)$ & 0.826 \\
\hline \multicolumn{5}{|l|}{ Cardiovascular } \\
\hline Hypertension & $3,445(45.1)$ & $2,840(44.0)$ & $605(51.1)$ & 0.048 \\
\hline Hyperlipidemia & $4,535(59.4)$ & $3,740(58.0)$ & $795(67.1)$ & 0.007 \\
\hline Angina & $170(2.2)$ & $140(2.2)$ & $30(2.5)$ & 0.742 \\
\hline Coronary artery disease & $5,090(66.7)$ & $4,370(67.8)$ & $720(60.8)$ & 0.043 \\
\hline Congestive heart failure & $130(1.7)$ & $110(1.7)$ & $20(1.7)$ & 0.984 \\
\hline Cardiogenic shock & $305(4.0)$ & $230(3.6)$ & $75(6.3)$ & 0.098 \\
\hline Endocarditis & $1,320(17.3)$ & $1,165(18.1)$ & $155(13.1)$ & 0.042 \\
\hline Pacemaker & $270(3.5)$ & $245(3.8)$ & $25(2.1)$ & 0.117 \\
\hline AICD & $205(2.7)$ & $185(2.9)$ & $20(1.7)$ & 0.219 \\
\hline CABG & $1,655(21.7)$ & $1,440(22.3)$ & $215(18.1)$ & 0.131 \\
\hline PCI & $1,320(17.3)$ & $1,155(17.9)$ & $165(13.9)$ & 0.111 \\
\hline Chronic pulmonary disease & $2,575(33.7)$ & $2,170(33.6)$ & $405(34.2)$ & 0.874 \\
\hline Peripheral vascular disease & $2,385(31.2)$ & $1,925(29.8)$ & $460(38.8)$ & 0.009 \\
\hline \multicolumn{5}{|l|}{ Renal } \\
\hline Chronic renal insufficiency/failure & $2,645(34.6)$ & $2,300(35.7)$ & $345(29.1)$ & 0.044 \\
\hline Hemodialysis & $100(1.3)$ & $100(1.6)$ & $0(0.0)$ & $<0.001$ \\
\hline Chronic liver disease & $145(1.9)$ & $>10(-)$ & $\leq 10(-)$ & 0.082 \\
\hline Diabetes & $2,210(28.9)$ & $1,865(28.9)$ & $345(29.1)$ & 0.951 \\
\hline \multicolumn{5}{|l|}{ Hematologic } \\
\hline Anemia & $2,000(26.2)$ & $1,740(27.0)$ & $260(21.9)$ & 0.090 \\
\hline Coagulopathy & $1,890(24.8)$ & $1,575(24.4)$ & $315(26.6)$ & 0.487 \\
\hline \multicolumn{5}{|l|}{ Constitutional } \\
\hline Frailty & $315(4.1)$ & $280(4.3)$ & $35(3.0)$ & 0.263 \\
\hline Overweight/obesity & $1,040(13.6)$ & $895(13.9)$ & $145(12.2)$ & 0.483 \\
\hline
\end{tabular}

Data are presented as $\mathrm{n}(\%)$ unless otherwise noted. Frequencies $\leq 10$ may not be disclosed as per the NIS data-use agreement. AICD = Automatic implantable cardioverter-defibrillator; PCI = percutaneous coronary intervention; TIA = transient ischemic attack. ${ }^{\text {a }}$ TA vs. TF.

$3,900)$ of the patients were male. The most common cardiovascular risk factors included coronary artery disease $(66.7 \%, \mathrm{n}=5,090)$, hyperlipidemia $(59.4 \%, \mathrm{n}=4,535)$ and hypertension $(45.1 \%, \mathrm{n}=3,445)$. Rates of chronic pulmonary disease, peripheral vascular disease and chronic re- nal insufficiency were each in excess of $30 \%(33.7 \%, \mathrm{n}=$ $2,575 ; 31.2 \%, \mathrm{n}=2,385 ; 34.6 \%, \mathrm{n}=2,645)$. Almost $30 \%$ of the patients $(\mathrm{n}=2,210)$ had diabetes, while anemia $(\mathrm{n}=$ $2,000)$ and coagulopathy $(\mathrm{n}=1,890)$ were comorbid in approximately $25 \%$ of patients. Upwards of $20 \%$ ( $n=$ 
Table 2. Hospital characteristics and procedural volumes

\begin{tabular}{|c|c|c|c|c|}
\hline & $\begin{array}{l}\text { Total } \\
(\mathrm{n}=7,635)\end{array}$ & $\begin{array}{l}\text { TF } \\
(n=6,450)\end{array}$ & $\begin{array}{l}\text { TA } \\
(n=1,185)\end{array}$ & $\mathrm{p}$ value $^{\mathrm{a}}$ \\
\hline \multicolumn{5}{|l|}{ Hospital characteristics } \\
\hline \multicolumn{4}{|l|}{ Bed size ${ }^{\mathrm{b}}$} & 0.983 \\
\hline Small & $340(4.5)$ & $285(4.4)$ & $55(4.6)$ & \\
\hline Medium & $1,050(13.8)$ & $890(13.8)$ & $160(13.5)$ & \\
\hline Large & $6,245(81.8)$ & $5,275(81.8)$ & $970(81.9)$ & \\
\hline \multicolumn{4}{|l|}{ Setting ${ }^{\mathrm{c}}$} & 0.137 \\
\hline Urban (nonteaching) or rural & $890(11.7)$ & $715(11.1)$ & $175(14.8)$ & \\
\hline Urban (teaching) & $6,745(88.3)$ & $5,735(88.9)$ & $1,010(85.2)$ & \\
\hline \multicolumn{4}{|l|}{ Region } & 0.593 \\
\hline Northeast & $1,725(22.6)$ & $1,495(23.2)$ & $230(19.4)$ & \\
\hline Midwest & $1,680(22.0)$ & $1,415(21.9)$ & $265(22.4)$ & \\
\hline South & $2,715(35.6)$ & $2,280(35.3)$ & $435(36.7)$ & \\
\hline West & $1,515(19.8)$ & $1,260(19.5)$ & $255(21.5)$ & \\
\hline \multicolumn{5}{|l|}{ TAVR volumes } \\
\hline Median annual TAVR cases/hospital (IQR) & $50(30-95)$ & $50(30-95)$ & $50(20-125)$ & 0.088 \\
\hline \multicolumn{4}{|c|}{ Distribution of TAVRs across hospitals performing } & $<0.001$ \\
\hline$<20$ TAVRs (123 centers) & $875(11.5)$ & $615(9.5)$ & $260(21.9)$ & \\
\hline 20-39 TAVRs (69 centers) & $1,780(23.3)$ & $1,565(24.3)$ & $215(18.1)$ & \\
\hline 40-59 TAVRs (32 centers) & $1,460(19.1)$ & $1,300(20.2)$ & $160(13.5)$ & \\
\hline$\geq 60$ TAVRs ( 34 centers $)$ & $3,520(46.1)$ & $2,970(46.0)$ & $550(46.5)$ & \\
\hline
\end{tabular}

Data presented as n (\%) unless otherwise noted. ${ }^{\mathrm{a}} \mathrm{TA}$ vs. TF. ${ }^{\mathrm{b}}$ See online suppl. appendix table 2 for bed size definitions. ${ }^{c}$ See online suppl. appendix table 3 for hospital setting definitions.

$1,655)$ of the patients had previously undergone coronary artery bypass grafting (CABG) and nearly $14 \%(\mathrm{n}=1,040)$ were overweight or obese.

\section{Hospital Characteristics and Procedural Volumes}

The majority $(81.8 \%, \mathrm{n}=6,245)$ of patients underwent TAVR at large hospitals and almost $90 \%(\mathrm{n}=6,745)$ received their TAVRs at urban teaching centers [table 2; online suppl. appendix tables 2 (hospital bed size) and 3 (setting)]. Of the 258 hospitals that performed $>7,600$ TAVRs included in this study, the median number of annual TAVR cases per center was 50 (IQR 30-95). The plurality $(46.1 \%, \mathrm{n}=3,520)$ of TAVRs were performed in the highest-volume' centers, i.e. in hospitals that performed at least triple the number of TAVRs mandated by CMS for reimbursement.

\section{Operative Details and Short-Term Outcomes}

Table 3 lists the operative details and short-term (i.e. inhospital) outcomes of the entire study cohort. The majority of patients $(84.5 \%, \mathrm{n}=6,450)$ underwent TF TAVR; the balance $(15.5 \%, \mathrm{n}=1,185)$ was treated with the TA approach. Although $30.4 \%(\mathrm{n}=360)$ of TA cases were performed on-pump, only $4.1 \%(\mathrm{n}=265)$ of the TF TAVRs required CPB $(\mathrm{p}<0.001)$.

Short-term mortality for the total cohort was 5.0\% $(\mathrm{n}=380)$; inhospital mortality did not differ significantly between patients receiving TF $(4.7 \%, \mathrm{n}=305)$ and $\mathrm{TA}$ TAVR $(6.3 \%, \mathrm{n}=75) ; \mathrm{p}=0.344$. As further outlined in table 3, 72.0\% $(\mathrm{n}=5,495)$ of all patients experienced some type of morbidity. Procedure-related complications occurred in $24.7 \%(n=1,885)$ of the TAVR patients. Even though the frequencies of overall procedural complications were equal for TF and TA TAVRs $(24.6 \%, \mathrm{n}=1,585$ vs. $25.3 \%, \mathrm{n}=300$, respectively, $\mathrm{p}=0.809$ ), the rates of several individual procedure-related complications did differ. Accidental punctures or lacerations, hematomas and other peripheral vascular procedural complications affected significantly more TF than TA patients $(3.4 \%$, $\mathrm{n}=220$ vs. $1.3 \%, \mathrm{n}=15, \mathrm{p}=0.016 ; 6.4 \%, \mathrm{n}=410$ vs. $3.0 \%$, $\mathrm{n}=35, \mathrm{p}=0.009$; and $\mathrm{n}>10$ vs. $\mathrm{n} \leq 10, \mathrm{p}=0.006)$. Aortic injuries and other nonspecified procedure-related cardiac complications were more common in the TA cases $(5.9 \%, \mathrm{n}=70$ vs. $2.4 \%, \mathrm{n}=155, \mathrm{p}=0.029$ and $13.9 \%, \mathrm{n}=$ 165 vs. $7.0 \%, \mathrm{n}=450, \mathrm{p}=0.004)$. 
Table 3. Operative details and short-term (i.e. inhospital) outcomes

\begin{tabular}{|c|c|c|c|c|}
\hline \multicolumn{5}{|l|}{ Operative details } \\
\hline CBP & $625(8.2)$ & $265(4.1)$ & $360(30.4)$ & $<0.001$ \\
\hline IABP & $160(2.1)$ & $110(1.7)$ & $50(4.2)$ & 0.065 \\
\hline \multicolumn{5}{|l|}{ Short-term outcomes } \\
\hline Any complication & $5,495(72.0)$ & $4,585(71.1)$ & $910(76.8)$ & 0.060 \\
\hline Procedural & $1,885(24.7)$ & $1,585(24.6)$ & $300(25.3)$ & 0.809 \\
\hline Valve/device complication & $280(3.7)$ & $245(3.8)$ & $35(3.0)$ & 0.490 \\
\hline Accidental puncture/laceration complicating surgery & $235(3.1)$ & $220(3.4)$ & $15(1.3)$ & 0.016 \\
\hline Bleeding complicating procedure & $295(3.9)$ & $230(3.6)$ & $65(5.5)$ & 0.222 \\
\hline Hematoma complicating procedure & $445(5.8)$ & $410(6.4)$ & $35(3.0)$ & 0.009 \\
\hline Cardiovascular & $4,140(54.2)$ & $3,425(53.1)$ & $715(60.3)$ & 0.038 \\
\hline Supraventricular arrhythmia & $3,505(45.9)$ & $2,890(44.8)$ & $615(51.9)$ & 0.046 \\
\hline Atrioventricular block & $155(2.0)$ & $120(1.9)$ & $35(3.0)$ & 0.348 \\
\hline Pacemaker insertion & $650(8.5)$ & $575(8.9)$ & $75(6.3)$ & 0.145 \\
\hline Postoperative shock & $165(2.2)$ & $120(1.9)$ & $45(3.8)$ & 0.137 \\
\hline Myocardial infarction & $295(3.9)$ & $235(3.6)$ & $60(5.1)$ & 0.350 \\
\hline Pulmonary embolism & $15(0.2)$ & $\leq 10(-)$ & $\leq 10(-)$ & 0.540 \\
\hline Mural/ventricular aneurysm & $\leq 10(-)$ & $\leq 10(-)$ & $0(0.0)$ & 0.318 \\
\hline Deep vein thrombosis & $75(1.0)$ & $>10(-)$ & $\leq 10(-)$ & 0.803 \\
\hline Pulmonary & $1,670(21.9)$ & $1,225(19.0)$ & $445(37.6)$ & $<0.001$ \\
\hline Postoperative acute pneumothorax & $60(0.8)$ & $>10(-)$ & $\leq 10(-)$ & 0.915 \\
\hline Wound infection & $50(0.7)$ & $>10(-)$ & $\leq 10(-)$ & 0.724 \\
\hline Urinary tract infection & $760(10.0)$ & $650(10.1)$ & $110(9.3)$ & 0.700 \\
\hline Median length of stay, days (IQR) & $6(4-10)$ & $6(4-10)$ & $7(5-11)$ & $<0.001$ \\
\hline Discharge status & & & & 0.293 \\
\hline Routine & $2,480(32.5)$ & $2,140(33.2)$ & $340(28.7)$ & \\
\hline Nonroutine & $4,775(62.5)$ & $4,005(62.1)$ & $770(65.0)$ & \\
\hline Deceased & $380(5.0)$ & $305(4.7)$ & $75(6.3)$ & \\
\hline
\end{tabular}

Data are presented as $\mathrm{n}(\%)$ unless otherwise noted. Frequencies $\leq 10$ may not be disclosed as per the NIS data-use agreement.

a TA vs. TF. ${ }^{b}$ Some patients had $>1$ particular morbidity within the same grouping of complications.

Many patients experienced cardiovascular complications $(54.2 \%, \mathrm{n}=4,140)$, with supraventricular arrhythmias occurring in $45.9 \%(\mathrm{n}=3,505)$ of patients overall; these were more common after TA TAVR than TF TAVR $(51.9 \%, \mathrm{n}=615$ vs. $44.8 \%, \mathrm{n}=2,890, \mathrm{p}=0.046) .3 .9 \%$ $(\mathrm{n}=295)$ of TAVR patients had myocardial infarctions, $1.4 \%(\mathrm{n}=105)$ suffered a stroke and $8.5 \%(\mathrm{n}=650)$ required permanent pacemaker insertion; none of these rates differed significantly between the TF and TA TAVR recipients. Pulmonary complications were noted in $21.9 \%$ 
Fig. 1. Unadjusted overall short-term (i.e. inhospital) mortality (a) and morbidity (b) from TAVR, stratified by hospital experience.

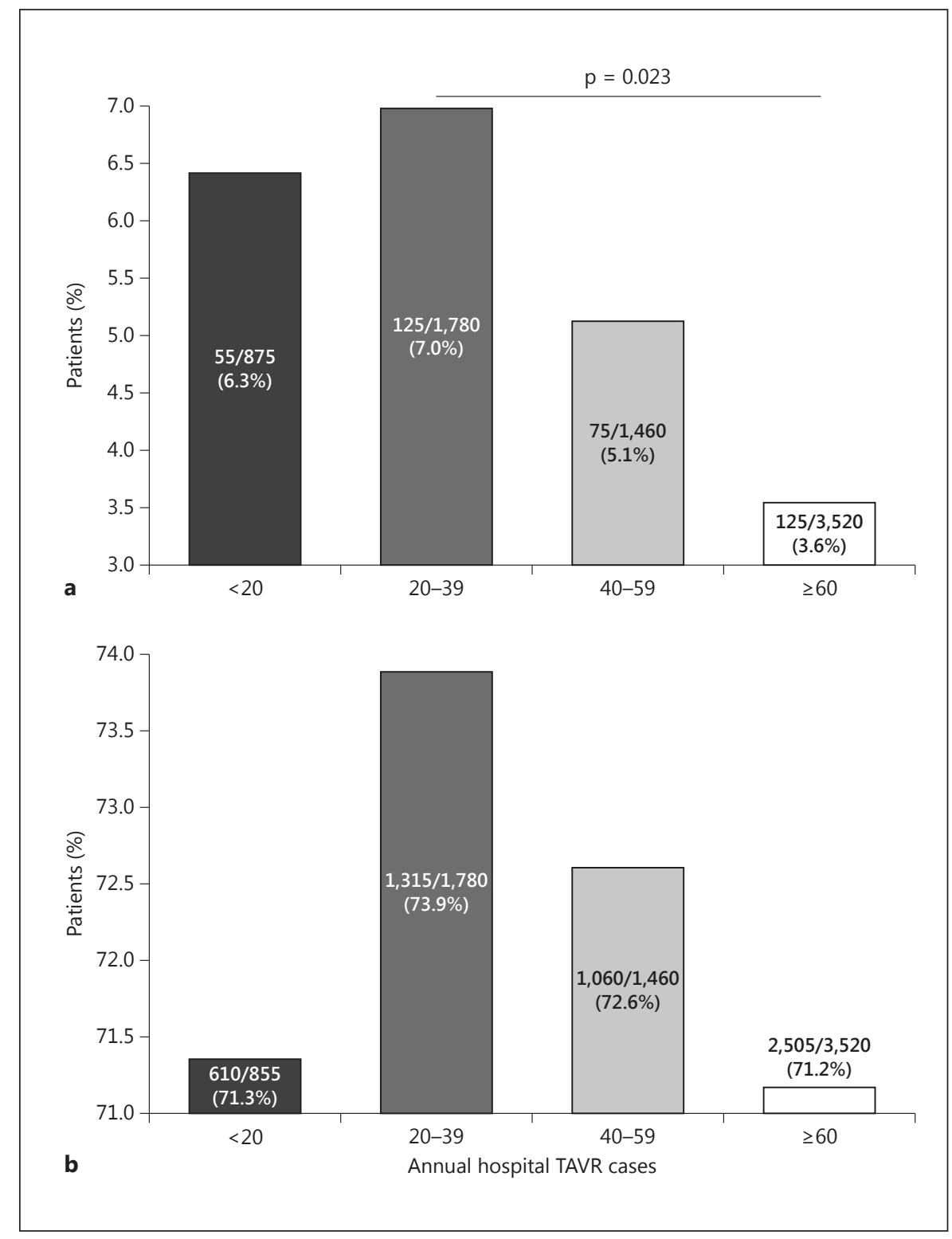

$(\mathrm{n}=1,670)$ of all patients and were more frequent after TA TAVR than after TF TAVR $(37.6 \%, \mathrm{n}=445$ vs. $19.0 \%$, $\mathrm{n}=1,225, \mathrm{p}<0.001) .12 .7 \%(\mathrm{n}=150)$ of the TA patients required mechanical ventilation compared to $6.5 \%(\mathrm{n}=$ $420)$ of the TF recipients ( $\mathrm{p}=0.007)$.

The overall median length of stay was 6 days (IQR 4-10 days); the median TA TAVR hospitalization was 1 day longer than that for TF TAVR (7 days, IQR 5-11 days vs. 6 days, IQR $4-10$ days, respectively, $\mathrm{p}<0.001$ ). Routine discharge (i.e. home, not against-medical-advice and not to any other facility) was achieved for $32.5 \%$ ( $\mathrm{n}=$ $2,480)$ of all patients.

National Analysis of TAVR Outcomes

\section{Volume-Outcome Relationships}

In order to assess for possible relationships between hospital TAVR experience and outcomes, we stratified the overall rates of unadjusted short-term mortality and morbidity by the TAVR volumes of the different centers (fig. 1). Mortality following TAVR at hospitals performing at least the minimum but no more than double the number of procedures required by CMS (i.e. 20-39 TAVRs) was nearly twice the mortality observed for the highest-volume centers ( 7.0 vs. $3.6 \%$, respectively, $\mathrm{p}=$ $0.023)$. There were no significant differences among the stratified hospitals with respect to overall morbidity. 
Table 4. Univariable and multivariable predictors of short-term (i.e. inhospital) mortality

\begin{tabular}{|c|c|c|c|c|}
\hline & \multicolumn{2}{|l|}{ Univariable } & \multicolumn{2}{|l|}{ Multivariable } \\
\hline & OR (95\% CI) & $\mathrm{p}$ value & OR (95\% CI) & $\mathrm{p}$ value \\
\hline \multicolumn{5}{|l|}{ Demographics } \\
\hline Age (continuous) & $1.02(0.99-1.06)$ & 0.129 & $1.05(1.01-1.09)$ & 0.006 \\
\hline \multicolumn{5}{|l|}{ Gender } \\
\hline Male & Reference & & & \\
\hline Female & $1.11(0.70-1.76)$ & 0.668 & & \\
\hline \multicolumn{5}{|l|}{ Race } \\
\hline White & Reference & & & \\
\hline Non-White & $0.97(0.50-1.87)$ & 0.917 & & \\
\hline \multicolumn{5}{|l|}{ Comorbidities } \\
\hline Stroke/TIA & $0.92(0.45-1.89)$ & 0.830 & & \\
\hline Hypertension & $0.48(0.29-0.80)$ & 0.004 & $0.50(0.25-0.98)$ & 0.044 \\
\hline Hyperlipidemia & $0.63(0.40-1.01)$ & 0.053 & $0.73(0.44-1.20)$ & 0.217 \\
\hline Coronary artery disease & $1.02(0.62-1.67)$ & 0.934 & & \\
\hline Endocarditis & $1.90(1.13-3.20)$ & 0.016 & $1.54(0.83-2.87)$ & 0.174 \\
\hline CABG & $0.60(0.31-1.15)$ & 0.122 & $0.63(0.33-1.19)$ & 0.158 \\
\hline PCI & $0.63(0.31-1.28)$ & 0.202 & $0.82(0.41-1.65)$ & 0.581 \\
\hline Chronic pulmonary disease & $0.96(0.59-1.57)$ & 0.875 & & \\
\hline Peripheral vascular disease & $1.30(0.81-2.10)$ & 0.281 & & \\
\hline Chronic renal insufficiency/failure & $1.48(0.93-2.36)$ & 0.101 & $1.00(0.53-1.88)$ & 0.996 \\
\hline Chronic liver disease & $0.68(0.09-5.06)$ & 0.704 & & \\
\hline Diabetes & $0.64(0.37-1.13)$ & 0.123 & $0.81(0.46-1.43)$ & 0.468 \\
\hline Anemia & $1.32(0.80-2.17)$ & 0.275 & & \\
\hline Coagulopathy & $1.84(1.13-2.97)$ & 0.014 & $1.55(0.90-2.67)$ & 0.116 \\
\hline Overweight/obesity & $0.43(0.17-1.09)$ & 0.074 & $0.64(0.26-1.61)$ & 0.344 \\
\hline \multicolumn{5}{|l|}{ Hospital characteristics and volumes } \\
\hline \multicolumn{5}{|l|}{ Setting ${ }^{\mathrm{a}}$} \\
\hline Teaching & Reference & & & \\
\hline Nonteaching & $0.89(0.42-1.88)$ & 0.753 & & \\
\hline Annual TAVR cases (continuous) & $0.99(1.00-1.00)$ & 0.028 & $1.00(0.99-1.00)$ & 0.111 \\
\hline Annual SAVR cases (continuous) & $1.00(0.99-1.00)$ & 0.122 & $1.00(0.99-1.00)$ & 0.882 \\
\hline \multicolumn{5}{|l|}{ Procedural } \\
\hline \multicolumn{5}{|l|}{ Approach } \\
\hline TF & Reference & & & \\
\hline TA & $1.36(0.76-2.44)$ & 0.299 & & \\
\hline CBP & $3.58(2.02-6.37)$ & $<0.001$ & $3.83(1.84-7.98)$ & $<0.001$ \\
\hline IABP & $15.55(7.35-32.93)$ & $<0.001$ & $19.52(6.97-54.70)$ & $<0.001$ \\
\hline
\end{tabular}

PCI = Percutaneous coronary intervention; SAVR = surgical aortic valve replacement; TIA = transient ischemic attack. ${ }^{a}$ See online suppl. appendix table 3 for hospital setting definitions.

\section{Predictors of Mortality and Morbidity}

Univariable and multivariable logistic regression analyses were then performed to evaluate whether hospital experience or other demographic, comorbidity and procedural variables were predictive of short-term (i.e. inhospital) mortality or morbidity.

Although comorbid hypertension, endocarditis and coagulopathy were found to be univariable predictors of mortality [odds ratio (OR) $0.48,95 \%$ confidence interval (CI) $0.29-0.80, \mathrm{p}=0.004$; OR 1.90, 95\% CI 1.13-3.20, $\mathrm{p}=0.016$ and OR $1.84,95 \%$ CI 1.13-2.97, $\mathrm{p}=0.014]$, only hypertension was significantly predictive of death on multivariable analysis (OR 0.50, 95\% CI 0.25-0.98, $\mathrm{p}=0.044$; table 4 ). Annual hospital TAVR volume was slightly protective against mortality when treated as a continuous variable in univariable regression (OR 0.99, 
Table 5. Univariable and multivariable predictors of short-term (i.e. inhospital) morbidity

\begin{tabular}{|c|c|c|c|c|}
\hline & \multicolumn{2}{|l|}{ Univariable } & \multicolumn{2}{|l|}{ Multivariable } \\
\hline & OR $(95 \% \mathrm{CI})$ & $\mathrm{p}$ value & OR $(95 \% \mathrm{CI})$ & $\mathrm{p}$ value \\
\hline \multicolumn{5}{|l|}{ Demographics } \\
\hline Age (continuous) & $1.01(0.99-1.02)$ & 0.351 & & \\
\hline \multicolumn{5}{|l|}{ Gender } \\
\hline Male & Reference & & & \\
\hline Female & $1.12(0.89-1.39)$ & 0.340 & & \\
\hline \multicolumn{5}{|l|}{ Race } \\
\hline White & Reference & & & \\
\hline Non-White & $0.98(0.72-1.34)$ & 0.916 & & \\
\hline \multicolumn{5}{|l|}{ Comorbidities } \\
\hline Stroke/TIA & $1.20(0.85-1.70)$ & 0.297 & & \\
\hline Hypertension & $0.72(0.58-0.90)$ & 0.004 & $0.74(0.54-1.01)$ & 0.060 \\
\hline Hyperlipidemia & $0.78(0.62-0.99)$ & 0.039 & $0.83(0.65-1.05)$ & 0.114 \\
\hline Coronary artery disease & $1.08(0.85-1.37)$ & 0.519 & & \\
\hline Endocarditis & $1.36(1.00-1.86)$ & 0.051 & $1.35(0.99-1.86)$ & 0.062 \\
\hline CABG & $0.91(0.69-1.18)$ & 0.470 & & \\
\hline PCI & $0.96(0.71-1.28)$ & 0.763 & & \\
\hline Chronic pulmonary disease & $1.18(0.93-1.50)$ & 0.172 & $1.22(0.95-1.55)$ & 0.113 \\
\hline Peripheral vascular disease & $1.18(0.92-1.51)$ & 0.189 & $1.13(0.88-1.45)$ & 0.350 \\
\hline Chronic renal insufficiency/failure & $1.18(0.93-1.49)$ & 0.178 & $0.89(0.64-1.24)$ & 0.502 \\
\hline Chronic liver disease & $1.02(0.45-2.33)$ & 0.957 & & \\
\hline Diabetes & $0.85(0.67-1.09)$ & 0.204 & $0.88(0.69-1.13)$ & 0.315 \\
\hline Anemia & $1.28(0.98-1.66)$ & 0.068 & $1.23(0.94-1.61)$ & 0.124 \\
\hline Coagulopathy & $1.56(1.18-2.05)$ & 0.002 & $1.50(1.14-1.99)$ & 0.004 \\
\hline Overweight/obesity & $0.93(0.67-1.28)$ & 0.654 & & \\
\hline
\end{tabular}

\begin{tabular}{lll}
\hline Hospital characteristics and volumes & & \\
Setting $^{\mathrm{a}}$ & & \\
$\quad$ Teaching & Reference & \\
Nonteaching & $0.85(0.61-1.20)$ & 0.365 \\
Annual TAVR cases (continuous) & $1.00(0.99-1.00)$ & 0.947 \\
Annual SAVR cases (continuous) & $1.00(0.99-1.00)$ & 0.914 \\
\hline Procedural & &
\end{tabular}

\section{Procedural}

Approach

$\begin{array}{cllll}\text { TF } & \text { Reference } & & & \\ \text { TA } & 1.35(0.97-1.86) & 0.073 & 1.29(0.92-1.81) & 0.147 \\ \text { CBP } & 1.45(0.93-2.26) & 0.097 & 1.32(0.84-2.08) & 0.227 \\ \text { IABP } & 5.98(1.42-25.16) & 0.015 & 5.30(1.27-22.17) & 0.022\end{array}$

PCI = Percutaneous coronary intervention; SAVR = surgical aortic valve replacement; TIA = transient ischemic attack. ${ }^{a}$ See online suppl. appendix table 3 for hospital setting definitions.

95\% CI 1.00-1.00, $\mathrm{p}=0.028)$ but was not predictive upon multivariable analysis. There were no significant predictive associations between centers' annual surgical aortic valve replacement caseloads and short-term mortality following TAVR (table 4). Similarly, TAVR approach (i.e. TF vs. TA) was not predictive of mortality. Utilization of either CPB or an IABP was highly predictive of post-TAVR mortality (OR 3.83, 95\% CI $1.84-$
7.98, $\mathrm{p}<0.001$ and OR 19.52, 95\% CI 6.97-54.70, $\mathrm{p}<$ $0.001)$.

Preoperative hypertension and hyperlipidemia were protective against overall morbidity in the univariable analysis (OR $0.72,95 \%$ CI $0.58-0.90, \mathrm{p}=0.004$ and OR $0.78,95 \%$ CI $0.62-0.99, \mathrm{p}=0.039$ ) but not in multivariable analyses (table 5). Comorbid coagulopathy, however, was found to be a significant multivariable predictor of 
Table 6. Comparisons of short-term (i.e. inhospital) outcomes from the NIS to findings from previous studies

\begin{tabular}{|c|c|c|c|c|c|c|c|c|c|}
\hline & \multicolumn{3}{|l|}{ NIS } & \multicolumn{3}{|c|}{ STS/ACC TVT [5] } & \multicolumn{3}{|l|}{ GARY [7] } \\
\hline & $\begin{array}{l}\text { Total } \\
(\mathrm{n}=7,635)\end{array}$ & $\begin{array}{l}\mathrm{TF} \\
(\mathrm{n}=6,450)\end{array}$ & $\begin{array}{l}\text { TA } \\
(\mathrm{n}=1,185)\end{array}$ & $\begin{array}{l}\text { Total } \\
(\mathrm{n}=7,710)\end{array}$ & $\begin{array}{l}\mathrm{TF} \\
(\mathrm{n}=4,972)\end{array}$ & $\begin{array}{l}\text { TA } \\
(\mathrm{n}=2,738)\end{array}$ & $\begin{array}{l}\text { Total } \\
(\mathrm{n}=3,875)\end{array}$ & $\begin{array}{l}\text { TF } \\
(\mathrm{n}=2,694)\end{array}$ & $\begin{array}{l}\text { TA } \\
(\mathrm{n}=1,181)\end{array}$ \\
\hline Mortality & $380(5.0)$ & $305(4.7)$ & $75(6.3)$ & $427(5.5)$ & $207(4.2)$ & $220(8.0)$ & $229(5.9)$ & $138(5.1)$ & $91(7.7)$ \\
\hline Stroke & $105(1.4)$ & $80(1.2)$ & $25(2.1)$ & $156(2.0)$ & $106(2.1)$ & $50(1.8)$ & $68(1.8)$ & 43 (1.6) & $25(2.1)$ \\
\hline
\end{tabular}

Data are presented as n (\%). GARY = German Aortic Valve Registry.

short-term morbidity after TAVR (OR 1.50, 95\% CI 1.14$1.99, \mathrm{p}=0.004)$ as was requiring an IABP (OR 5.30, 95\% CI 1.27-22.17, $\mathrm{p}=0.022$ ).

\section{Discussion}

\section{Principal Findings}

To date, this study represents the largest examination of short-term TAVR outcomes using non-self-reported data, i.e. data that were independent from other institutional or society registries and clinical trials. In leveraging the NIS, we demonstrated a nationwide, inhospital mortality rate of 5.0\% for patients undergoing TAVR. Stroke was observed in $1.4 \%$ of patients, but overall procedurerelated morbidity occurred in almost $25 \%$ of cases. While unadjusted data suggested a possible association between hospital TAVR volumes and short-term mortality, no such volume-outcome relationships emerged upon more rigorous multivariable regression analyses.

\section{Results in Context}

The short-term results we describe above largely mirror those reported in several other large-scale, postapproval TAVR studies (table 6). The most recent analysis of the STS/ACC TVT Registry, for example, documented rates for inhospital mortality and stroke of 5.2 and $1.9 \%$, respectively [10]. In its report immediately prior to that one, it stratified these short-term outcomes on the basis of TAVR approach [5]; here too, our findings closely match those of the Registry. The German Aortic Valve Registry identified inhospital mortality and stroke in 5.9 and $1.8 \%$ of its 3,875 TAVR patients, respectively [7]; our short-term TF and TA outcomes are in line with those from this registry. In this light, the results provided by the NIS (1) help to affirm the short-term safety of TAVR during the era of its commercialization and rapid expansion and (2) validate the findings of these TAVR-specific databases.

\section{Prognostic Factors: Patient- and Hospital-Level Variables}

Although the primary objective of our study was to describe the contemporary, short-term outcomes for patients undergoing TAVR, we also sought to identify risk factors for mortality and/or morbidity following this procedure. In so doing, we found that requiring CPB or an IABP were each significantly predictive of inhospital mortality after TAVR (table 4). Neither of these modes of support is routinely needed during TAVR, so it is not surprising that both emerged as markers of mortality, given that they were likely utilized in the emergency of intraoperative cardiovascular collapse. Interestingly, despite the fact that we assessed many of the same variables, the TVT Registry group identified increasing age, male gender, severe chronic obstructive pulmonary disease, renal failure, atrial fibrillation and non-TF access as additional predictors of mortality following TAVR, but it must be noted that their analysis was at the 1-year time point [10].

An important distinction of our study is that we further considered whether hospital experience with TAVR played any role in determining the short-term outcomes of patients undergoing this type of valve surgery. Perhaps counterintuitively, the results of our multivariable analyses demonstrated that, when treated as a continuous variable, annual hospital TAVR volume was not predictive of inhospital mortality or morbidity, following TAVR (table 4, 5). One can speculate that, in 2012, despite commercialization, many TAVRs were still being performed at centers with considerable transcatheter experience given the participation by these hospitals in the original TAVR trials. It will therefore be intriguing to see the results of similar analyses when data for the subse- 
quent years become available from the NIS, as these will include more hospitals with new or evolving TAVR programs.

\section{Limitations}

There are several limitations to this study, most notably with respect to the type of data that are not collected by the NIS. The NIS is a powerful tool in that it provides us with reliable, independent, cross-sectional outcome statistics for very large numbers of patients; it does not, however, capture certain patient-level data of particular interest in TAVR. Data like valve brand/model, STS risk scores, frailty or functional-status measures (e.g. the Kansas City Cardiomyopathy Questionnaire or the 6-min walk test), New York Heart Association class, the presence of preoperative atrial fibrillation or postoperative new-onset arrhythmias, echocardiographic findings, operative times and transfusion requirements are all absent from the NIS. Not only would such information provide us with more detailed descriptions of our study cohort, inclusion of these data would be helpful in constructing more comprehensive predictive models of mortality and morbidity following TAVR.

Another limitation involves the data that are captured by the NIS; since the database relies on ICD-9-CM codes to describe the characteristics of patients and procedural outcomes, how these codes are defined (and interpreted by those feeding in the data) can hypothetically skew results. Most ICD-9-CM codes are not rigorously defined, so, for example, the exact complication constituting 'Hematoma Complicating Procedure' (code 998.12) for 1 patient in the NIS might differ from what was actually experienced by the next patient with that code. This may explain why we observed relatively high rates of proce- dural complications, and it ultimately underscores the utility of standardized definitions for clinical end points, like those set forth by the Valve Academic Research Consortium [18].

Even when certain outcomes of interest are represented by series of ICD-9-CM codes, these codes do not often provide the level of detail requisite for meaningful analyses. For instance, although we were able to describe rates of postoperative supraventricular arrhythmias, current ICD-9-CM codes do not distinguish between preexisting and new-onset rhythm disturbances. Hence, the overall $45.9 \%$ prevalence of supraventricular arrhythmias in our cohort (table 3) was likely inflated by the inclusion of patients who had these disturbances preoperatively as well.

\section{Conclusions}

Our analysis further documents the short-term safety of TAVR during an era notable for the commercialization of this technique, thereby independently corroborating the results of several contemporary TAVR registries. While inhospital mortality remains acceptably low, this study highlights an opportunity for additional improvements with regard to procedure-related complications. As updated datasets become available, investigations into hospital and provider experience may provide insights into heretofore unrecognized prognostic factors in TAVR.

\section{Conflict of Interest}

Dr. Salemi is a site co-PI for Edwards Lifesciences and he has served as a proctor and consultant for the company. The remaining authors have no disclosures.

\section{References}

1 Leon MB, Smith CR, Mack M, Miller DC, Moses JW, Svensson LG, et al: Transcatheter aortic-valve implantation for aortic stenosis in patients who cannot undergo surgery. $\mathrm{N}$ Engl J Med 2010;363:1597-1607.

2 Smith CR, Leon MB, Mack MJ, Miller DC, Moses JW, Svensson LG, et al: Transcatheter versus surgical aortic-valve replacement in high-risk patients. N Engl J Med 2011;364: 2187-2198.

3 Holmes DR Jr, Mack MJ, Kaul S, Agnihotri A, Alexander KP, Bailey SR, et al: 2012 ACCF/
AATS/SCAI/STS expert consensus document on transcatheter aortic valve replacement. J Am Coll Cardiol 2012;59:1200-1254.

4 Généreux P, Head SJ, Van Mieghem NM, Kodali S, Kirtane AJ, Xu K, et al: Clinical outcomes after transcatheter aortic valve replacement using valve academic research consortium definitions: a weighted meta-analysis of 3,519 patients from 16 studies. J Am Coll Cardiol 2012;59:2317-2326.

5 Mack MJ, Brennan JM, Brindis R, Carroll J, Edwards F, Grover F, et al: Outcomes follow- ing transcatheter aortic valve replacement in the United States. JAMA 2013;310:20692077.

6 Adams DH, Popma JJ, Reardon MJ, Yakubov SJ, Coselli JS, Deeb GM, et al: Transcatheter aortic-valve replacement with a self-expanding prosthesis. N Engl J Med 2014;370:17901798.

7 Hamm CW, Möllmann H, Holzhey D, Beckmann A, Veit C, Figulla HR, et al: The German Aortic Valve Registry (GARY): in-hospital outcome. Eur Heart J 2014;35:1588-1598. 
8 Department of Health and Human Services: Correspondence regarding premarket approval application for Edwards Sapien transcatheter heart valve. November 2, 2011. http://www.accessdata.fda.gov/cdrh_docs/ pdf10/p100041a.pdf (accessed March 16, 2015).

9 Department of Health and Human Services: Correspondence regarding premarket approval application for Edwards Sapien transcatheter heart valve and transapical and transfemoral accessories. October 19, 2012. http:// www.accessdata.fda.gov/cdrh_docs/pdf11/ P110021a.pdf (accessed March 16, 2015).

10 Holmes DR Jr, Brennan JM, Rumsfeld JS, Dai D, O'Brien SM, Vemulapalli S, et al: Clinical outcomes at 1 year following transcatheter aortic valve replacement. JAMA 2015;313: 1019-1028.
11 Overview of the National (Nationwide) Inpatient Sample (NIS). http://www.hcup-us. ahrq.gov/nisoverview.jsp (accessed March 5, 2015).

12 Birkmeyer JD, Siewers AE, Finlayson EV, Stukel TA, Lucas FL, Batista I, et al: Hospital volume and surgical mortality in the United States. N Engl J Med 2002;346:1128-1137.

13 Trivedi AN, Sequist TD, Ayanian JZ: Impact of hospital volume on racial disparities in cardiovascular procedure mortality. J Am Coll Cardiol 2006;47:417-424.

14 Fugate JE, Brinjikji W, Mandrekar JN, Cloft HJ, White RD, Wijdicks EF, et al: Post-cardiac arrest mortality is declining: a study of the US National Inpatient Sample 2001 to 2009. Circulation 2012;126:546-550.

15 Paul S, Jalbert J, Isaacs AJ, Altorki NK, Isom OW, Sedrakyan A: Comparative effectiveness of robotic-assisted vs. thoracoscopic lobectomy. Chest 2014;146:1505-1512.
16 Comorbidity software v3.7. http://www. hcup-us.ahrq.gov/toolssoftware/comorbidity/comorbidity.jsp (accessed January 24, 2015).

17 Centers for Medicare and Medicaid Services: National Coverage Determination (NCD) for Transcatheter Aortic Valve Replacement (TAVR) (20.32). http://www.cms.gov/medicare-coverage-database/details/ncd-details. aspx?NCDId=355 (accessed April 7, 2015).

18 Kappetein AP, Head SJ, Généreux P, Piazza $\mathrm{N}$, van Mieghem NM, Blackstone EH, et al: Updated standardized end point definitions for transcatheter aortic valve implantation: the Valve Academic Research Consortium-2 consensus document. J Thorac Cardiovasc Surg 2013;145:6-23. 